\title{
Seeking new pathways for HIV vaccine discovery
}

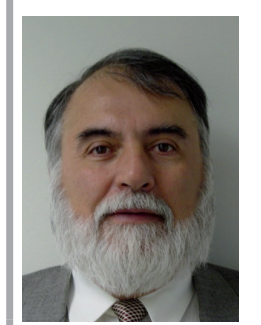

"...the substantial increase in antiretroviral treatment coverage has not resulted in the expected impact on the spread of the epidemic ... a prophylactic vaccine continues to be the potentially most costeffective approach to halt it."

Jorge Flores

Vaccine Research Program, Division of AIDS, National Institute of Allergy and Infectious Diseases, National Institutes of Health, Bethesda, MD 20892, USA = Tel.: +1 3014353758 = Fax: +1 3014023684 = jllores@niaid.nih.gov

Since the beginning of the HIV epidemic in the mid-1980s, over 60 million people have been infected with HIV worldwide and about half of them have died. The limited impact of implementing known preventive measures and the negative results of over 15 efficacy trials of microbicides, vaccines and other biomedical modalities offers little prospect for a prompt solution to this crisis. Fortunately, not all the news is bad, efficacy trials of circumcision have shown strong evidence of protecting men at risk through heterosexual sex. It is well established that HIV shedding drops significantly during antiretroviral therapy (ART) and should therefore diminish transmission; however, the substantial increase in antiretroviral treatment coverage has not resulted in the expected impact on the spread of the epidemic; it might take more intensive programs of frequent testing and early treatment to achieve the coverage required to see such an effect [1]. Currently, overall transmission rates are somewhat stable, though they have been curtailed in some countries; it is estimated that for every 1 million patients starting treatment, 2.7 million new infections occur [101]. Consequently, prevention continues to be regarded as the only viable way out of the epidemic, and a prophylactic vaccine continues to be the potentially most cost-effective approach to halt it.

The discovery of an HIV vaccine has eluded the strategies of traditional vaccine development and modern science. No vaccine candidates are on the horizon that address the most demanding requirements that such a vaccine needs to fulfill: interrupt transmission, abort early viral replication, prevent dissemination of the virus from the initial mucosal entry site, ameliorate progression of disease and prevent secondary transmission. The virus imposes many challenges that are to a large extent well understood: destruction of the immune system, integration in the host genome, latency with the ability to resurge, and an extreme ability to rapidly mutate and adapt. Effectively counteracting these properties has proved to be impossible thus far. No other infectious agent for which vaccines have been developed in the past presented such an array of challenges. Regrettably, all rational and empiric attempts used to develop previously successful vaccines have failed to yield a HIV vaccine. In addition, multiple novel and clever approaches have been tested with little success, although they have incrementally contributed to a large body of knowledge that could potentially lead to a more effective vaccine design. Topping the list of disappointments is the current lack of immunogens that can induce broadly reactive neutralizing antibodies to contain the immense variety of circulating HIV-1 strains. If such immunogens were available, infection might be prevented or at least limited before the virus has a chance to infect a critical mass of target cells and destroy the immune system. Two efficacy trials of envelope-based subunit vaccines developed by VaxGen designed to induce neutralizing antibodies, failed to prevent infection in spite of inducing a strong immune response to the homologous viruses from which the vaccines were derived, but not to heterologous strains [2]. Despite enormous efforts preceding and following those trials little progress has been made in designing immunogens that substantially improve cross-reactivity compared with those that failed.

Two key observations point to the possibility that exploiting the cellular arm of the immune system may lead, if not to the prevention of infection, at least to the control of disease progression and diminution of secondary transmission: first, during natural infection, the decline in viral load coincides with the development 
of a strong cytotoxic T lymphocyte (CTL) response, and second, depletion of CTLs in nonhuman primates (NHPs) vaccinated with simian immunodeficiency virus (SIV) vaccines that can exert partial control of their viral load, leads to obliteration of such control. Clinical observations in 'elite' controllers, long-term nonprogressors and exposed uninfected individuals support the role for CTLs in containing infection. Many experimental HIV vaccines have been able to induce anti-HIV or -SIV specific CTLs in NHPs, but only a few have shown enough promise in humans to warrant advanced development. Furthermore, the most advanced candidate that induced cellular immunity (the Merck HIV adenovirus-vectored vaccine) recently failed to afford protection in a large Phase II test of concept efficacy trial [3]. The field awaits the results of an additional efficacy trial of a combination of a T-cell based vaccine (a canarypox-HIV recombinant developed by Sanofi-Pasteur [Paris, France]) and the VaxGen subunit vaccine, which will be released towards the end of 2009.

\section{"...the sustained and vigorous effort \\ expended on the development and testing of HIV vaccines needs to continue..."}

It is clear from the product failures mentioned that the sustained and vigorous effort expended on the development and testing of HIV vaccines needs to continue, but also highlights the need to strengthen basic approaches to substantially expand the depth and breadth of the research to discover new leads, develop and test new hypotheses, and explore novel approaches. An HIV Vaccine Summit convened by the National Institute of Allergy and Infectious Diseases in 2008 concluded that the balance of research and development needs to tip towards more fundamental research [4]. This will require a concerted profound inquiry into scientific disciplines traditionally unexploited in vaccine research, the creation of nontraditional approaches to identify antigens and vaccine designs, and exploration of other 'nonvaccine' immune approaches to interrupt transmission, particularly those that could provide sustained protection with a single or few administrations.

In response to this need, the menu of programs from governments, foundations and research institutions to support development of a HIV vaccine and other measures to interrupt HIV transmission needs to be expanded in depth and breadth by promoting the identification and translation of new leads from basic research into vaccine design. Clearly, more intense work in some research areas will provide fruitful leads (e.g., understanding the early events in viral transmission, better knowledge of the immune system, new tools to analyze large databases, and so on), but important results might also come from research in contiguous and, to some extent, in two-step removed fields. In addition, sufficient space needs to be provided to allow for indirect discoveries, outside-the-box approaches, serendipity or even counterintuitive research that challenges established paradigms. The high risk of these tactics needs to be carefully balanced with continuous exploitation of the direct paths and logical extensions of current research, such that progress continues to be incremental with the hope of a burst of new knowledge that could translate into exponential advances.

\section{Direct paths to promote HIV vaccine discovery A better understanding of viral pathogenesis}

Thorough knowledge of the HIV virus and its pathogenesis has been accumulating exponentially thanks to the sustained efforts of unrelenting scientists and generous support from sponsors, to the extent that, in spite of its complexity, we know more about this virus than about any other pathogen. Nonetheless, we urgently need to respond to some essential questions critical for vaccine development, many of which originate from the research itself. Key among them is to address early events in mucosal transmission and factors that contribute to viral pathogenesis.

Recently, through intense efforts of the NIH-sponsored Center for HIV-AIDS Vaccine Immunology (CHAVI), a substantial number of 'bona fide' acute infections have been studied, including the characterization of the 'transmitted' viruses [5]. These viruses may exhibit unique biological properties and/or possess common antigenic targets to incorporate into a HIV vaccine; likewise, structural studies of their envelopes will help understand the structural conformations relevant to the induction of neutralizing antibodies, especially those with broad reactivity. Insight into the genetic evolution of the transmitted viruses over time, studied in conjunction with the kinetics and specificity of the antibody and T-cell responses in the context of the individual's MHC should shed light on the immune pressure mounted by the host and its effect on disease progression, illuminating vaccine discovery. 
Given the irreversible integration of the HIV genome into the human genome, a vaccine that prevents transmission should induce an effective memory response that is promptly evoked by an infectious challenge before integration occurs. It is not clear whether effector activities (antibodies and effector memory $\mathrm{T}$ cells) will be recalled to block the virus in a timely manner or whether the immune response will have to be programmed to be continuously present at vulnerable mucosal sites.

Studying the earliest events in HIV-1 transmission is virtually impossible in humans, and thus it has to rely on the pathogenic SIV challenge model in NHPs. Studies in NHPs have helped identify the initial target cells as $\mathrm{CD}^{+}{ }^{+} \mathrm{T}$ cells, macrophages and dendritic cells, though with a degree of controversy, since some cells to which the virus attaches may simply act as carriers while others are clearly substrates for replication. Attachment of the virus to local dendritic cells eventually leading to infection may occur as early as $20-30 \mathrm{~min}$ postinfection and virus replication takes place in the ensuing hours and days. Immune activation, even at this early stage, contributes to amplification of this phenomenon. From these founder populations, the virus and infected cells are exported to the draining lymph nodes and eventually to the circulation [6]. Multiple factors, including the innate immune response, may regulate viral replication at this early stage. Although postulated by some, but not demonstrated, transient subclinical mucosal infections might occur in humans. Perhaps abortive infections, if they indeed occur, are the product of insufficient seeding or of some control by early $\mathrm{T}$-cell responses. To what extent this initial replication can be aborted is at the core of HIV vaccine research. Certainly, it is less likely that once the virus reaches the gut-associated lymphoid tissue the progression of infection can be completely detained.

\section{"Although postulated by some, but \\ not demonstrated, transient subclinical \\ mucosal infections might occur in humans."}

The importance of immune activation in HIV infection was a subject of intense controversy until a few years ago. Its role in contributing to pathogenesis (rather than simply being a consequence of viral infection) is now supported by the observation that the attenuation of SIV infection observed in the natural host (sooty mangabee) is not associated with a lower level of viral replication or preservation of $\mathrm{CD}^{+} \mathrm{T}$ cells, but rather to the absence of an elevated T-cell activation level. On the other hand, rhesus macaques, when challenged with the same virus, experience increased T-cell activation. An increased permeability of the intestinal barrier to bacterial products appears to be responsible for the observed immune activation; this is supported by the observation that rhesus macaques exhibit high levels of circulating lipopolysaccharide during infection, while sooty mangabees do not. A single administration of lipopolysaccharide in sooty mangabees simulates the enteropathy seen in the rhesus macaques, resulting in a burst in viral load and $\mathrm{CD}^{+} \mathrm{DR}^{+}$ cells [7]. Unfortunately, in spite of the thorough recognition of the phenomenon of immune activation, little has been done in the field to attempt to prevent or counteract it.

"The importance of immune activation in HIV infection was a subject of intense controversy until a few years ago."

CHAVI studies have recently led to the recognition that during the earliest stages of infection release of enormous quantities of cell death factors, such as TNF-related apoptosis-inducing ligand (TRAIL), Fas ligand, TNF receptor type 2 and plasma microparticles, contribute to $\mathrm{CD}^{+}$ T-cell destruction and to immune suppression of T cells, macrophages and B cells, which could account for the poor immune responses observed from the beginning of the infection [8]. Some of those cells targeted by apoptotic factors are, of course, the ones that one would like to prime with a vaccine.

\section{Better understanding of the immune response}

Understanding the mechanisms of immune protection to HIV has been hampered by the difficulties in identifying early cases of infection, the interdependence between host genetics and HIV infection outcome, and the limited predictability of animal models. The immune response mounted by the host during infection has been well characterized, however, it is still not clear to what extent the various immune responses elicited by infection are directly able to control the infection. The potential beneficial role of antibody in HIV infection has been demonstrated in passive protection studies in NHPs as well as in studies of the neutralizing antibody responses to the virus in humans during infection, which although late in appearing, exert selective pressure causing the virus to mutate and escape. This belated response does not appear to be effective 
in controlling the virus, besides the specificity of the neutralizing antibody is relatively narrow early in infection [9], and only after a few years are broadly cross-reactive responses detected. As noted earlier, candidate vaccines thus far have only been able to induce limited breadth. Recently, information has emerged pointing to a potential inability of the immune system to generate cross-reactive neutralization responses due to either limitations in the B-cell repertoire, or the recognition of the critical viral determinants as 'self' leading to their removal. While chronic infection with continuous antigenic re-exposure of specific B cells in the germinal centers may eventually induce increased affinity and breadth through somatic mutations, the possibility of mimicking this with a vaccine may be limited.

The current explosion of knowledge of T cells and innate immunity offers countless possibilities to manipulate the immune response in multiple directions, from improvement in innate responses and consequent modulation of the primary adaptive responses to programming the character, functionality, migration and memory properties of the resulting HIV-specific $\mathrm{T}$ cells.

\section{"The current explosion of knowledge of $T$ cells and innate immunity offers countless possibilities to manipulate the immune response in multiple directions..."}

A worrisome challenge not previously recognized has been brought to light by the suspicion that the increased acquisition observed in a subgroup of volunteers in the trial of the Merck Adeno 5 vector-based vaccine may have been the consequence of vaccine-induced activation of preexisting adeno-specific cells [10], HIV-specific cells or even bystander cells. Theoretically, activation of $\mathrm{CD}^{+} \mathrm{T}$ cells, a desirable effect for a vaccine, might create a better substrate for the virus to grow. If so, how would one induce appropriate specific T-cell responses without increasing the chances of enhanced acquisition?

Accelerated progress in defining what immune responses are protective will require the development of novel in vitro systems and the application of multiple methodologies to broadly examine genomes, transcription products and proteins, and systems biology analysis tools that will provide additional leads to understand the behavior of the virus and identify potential new targets to control it. An example of such an application is the recent analysis of the responses to a well known, highly effective vaccine, the live-attenuated yellow fever vaccine [11].

\section{A broader approach to discovery}

HIV vaccine discovery needs to be defined in a broad sense as the generation, exploration and exploitation of scientific knowledge to identify new targets and new immune-based approaches to block HIV transmission and detain progression, applying established technologies but also making the most of new technologies developed for other purposes. This path requires profound and sustained surveillance and translation of current discoveries in cell biology, structural biology, virology, immunology and other disciplines. It also requires ample room for innovation, testing of outside-the-box approaches and enhanced analytical tools.

While clear paths focused on the discovery and design of appropriate antigens have been successful for the development of other vaccines, antigen discovery alone has proven to be insufficient to tackle the requirements to prevent $\mathrm{HIV}, \mathrm{TB}$, malaria and other infections. The recent explosion of our knowledge of innate immunity and growing understanding of adaptive immunity would potentially allow for the creation of new vaccine discovery paths. Perhaps the key ingredient in a discovery program is a thorough understanding of the pathogenesis of the infection and the host-microbe relationship, in particular the early events in transmission when a vaccine must act to prevent. Can such a multipronged approach comprising antigen discovery, immune response and pathogenesis research be systematized in a clear manner to accelerate discovery? Some would argue that science in general lacks proven principles to maximize the probabilities of discovery and that few efforts have been made to develop such principles [12]. Traditionally, scientists decide from their knowledge, intuition and principles imprinted during their training about the appropriate experimental approaches to test the key questions in the field based on hypotheses with varying levels of supporting evidence. They also take advantage of opportunities afforded by the availability of new knowledge, new techniques and new reagents; however, they lack validated principles to maximize the probability of discovery and enhance the chances that their accomplishments will have an impact in their field, especially on a targeted goal.

Discoveries are sometimes made by mere serendipity; all scientists are familiar with significant 'accidental' discoveries in medicine (Pasteur's discovery of microbial attenuation, Fleming's 
discovery of penicillin, Roentgen's discovery of X-rays). It is often said that discoveries are the product of keen observing eyes, but in most cases many decades of inquiry contribute to the moment when the pieces of the puzzle that initially appeared distant came together. An example of this in the vaccine field is the discovery that carbohydrate molecules when covalently conjugated to specific antigens significantly increase their antigenicity [13], a concept successfully applied to the development of typhoid, Haemophilus influenza $\mathrm{B}$ and other vaccines.

One 'proven' novel path to vaccine discovery is undoubtedly the 'reverse vaccinology' approach, a term coined by Rappuoli, and applied successfully by him and his colleagues to the development of a Neisseria meningitides vaccine and with persuasive promise for the development of several other bacterial vaccines [14]. Antigen discovery is at the core of this approach, which exploits the available genome sequence of a pathogen by applying multiple algorithms to predict and identify 'protective antigens' (surface exposure, glycosylation, HLA binding, and so on). A significant advance that complements this approach uses targeted mutagenesis to identify essential genes and tests vulnerabilities in pathogens to be targeted by a vaccine or an antimicrobial agent [15]. Translation of these approaches to the development of viral vaccines, and especially to an HIV vaccine has been of limited success. Although these approaches have allowed us to identify potential T-cell epitopes common to many viruses, and design 'consensus' sequence antigens to address the virus variability issue, they have fallen short for designing antigens that induce broadly cross-reactive neutralizing antibodies.

The drug industry uses the more direct discovery approach of exploiting combinatorial chemistry to synthesize and screen numerous compounds for the desired - and sometimes an unexpected - useful activity. Many argue that this process is inefficient, however, it is still one of the key paradigms of the pharmaceutical industry and more than a handful of notable drugs have been discovered in this way. It is difficult to imagine how a similar approach can be designed to test the myriad of ways in which the immune response can be tweaked. Perhaps the development of appropriate 'in vitro' vaccination methods would allow for an equivalent approach.

A rational approach to develop and identify the proper antigens, adjuvants or other stimulatory molecules more predictably for inclusion in a vaccine will require extensive knowledge of the complex mechanisms that regulate the immune response. Multiple 'discoveries' will have to be made in this area to allow for success. Luckily, multiple leads are continuously being unearthed, and undoubtedly some of them might eventually prove to be useful while others will uncover additional challenges not initially realized (Table 1). For example, the key to success may reside in stimulating innate immunity pathways to prime a certain type of response, program immune cells to mature and commit to certain functional, memory and migration phenotypes, or even downregulate normal repressor mechanisms. However, for HIV it is not known what features of the immune response will be adequate to afford protection. Addressing this will require knowledge that can only be obtained from optimal animal models of vaccination/challenge with appropriate vaccines as well as information from human studies of natural HIV infection and clinical vaccine trials that test for efficacy. Undoubtedly, a huge body of understanding is waiting to be unraveled by immunologists and HIV virologists. Analyzing and exploiting the myriad of complex interactions among the viral genes and proteins and the cell's machinery, and between the virus and the immune system may well be beyond human capacity and will have to be complemented by robust modeling approaches at all levels.

\footnotetext{
"Discoveries are sometimes made by mere serendipity; all scientists are familiar with significant 'accidental' discoveries in medicine..."
}

While classical scientific strategies are based on de-convoluting phenomena to analyze individual components in a linear way, it is clear that all cellular functions are the result of complex interactions; this is particularly true for the immune system, in which perturbing a single gene or protein will affect a wide range of functions with multiple retro-feedback interactions in multiple cell types. The upsurge of high-throughput technologies (genomics, proteomics, transcriptomics, and so on) allows the generation of large amounts of data, and at the same time poses a challenge to their analysis. Interpretation and mining has to rely on computer-based analysis of greater power than those available thus far, and in the future may be assisted by advances in the rapidly expanding world of artificial intelligence. To meet this challenge, it is necessary to accelerate the pace of systems biology approaches in which new conceptual frameworks requiring novel statistical 
Table 1. HIV vaccine development challenges.

\section{Historical challenges}

- Viral genetic and antigenic variability

- Refractoriness to neutralization

- Destruction of the immune system

- Integration and latency

- Multiple transmission routes

\section{Newly unearthed challenges}

- Vaccine-induced T-cell activation may increase the chances of infection

- Data from the STEP trial (efficacy of the Merck Adenovirus 5-vectored HIV vaccine) suggest that the vaccine might have enhanced susceptibility to acquisition of HIV in a subgroup of volunteers.

- The normal B-cell repertoire may not contain specificities that target conserved neutralization determinants in the virus. Are they removed by tolerance mechanisms? Can they be induced by a vaccine?

- The few broadly neutralizing antibody specificities isolated from infected individuals appear to require extensive somatic hypermutation of germline antibody genes.

- Can/should vaccine-induced T-cell activation be averted?

- T-cell activation has been shown to be a key pathogenic determinant, not just a consequence of infection.

- Given the narrow window for preventive vaccines to work, what is the appropriate balance of effector/central memory cells to induce with a vaccine?

- Nonhuman primates and acute infection studies in humans show that the eclipse phase of infection (between initial challenge and peak viral load) is very narrow - just a few days.

- The recall response to a vaccine may be compromised during early infection.

- Apoptotic factors generated very early in infection have a detrimental effect on macrophages, monocytes, CD4 ${ }^{+}$T cells and $B$ cells.

and computational methodologies are applied in an iterative recycling of experimental analysis, appropriate modeling and laboratory validation to improve not only data interpretation, but also the formation and testing of new hypotheses.

Genome-wide association studies, although nonhypothesis-driven, constitute an important component of a 'path to discovery' of a HIV vaccine. The power of the currently available technologies, especially of SNP analysis, has shown to be effective in identifying the genes responsible for many disorders. Its application in studies of HIV infection has led to the identification of at least three sets of genes associated with a better prognosis of disease progression [16].

A bit of anarchism in science might yield some success [17]. For example, experiments to test hypotheses that contradict well-confirmed theories and/or well established experimental results might lead the field to advances or better understanding of some phenomena. Because the consistency condition of science demands that every new hypothesis agrees with accepted theories, new ideas tend to be rejected, yet proliferation of numerous theories is beneficial to achieving progress in science. Scientists' familiarity with accepted theories might predispose them to view new ideas in a prejudicial manner; however, some scientific advances have been achieved when the prescriptive rules of science are 'violated'.
While at the individual level most scientists approach discovery as the search for that 'gold nugget', which requires thorough digging and better luck, the field as a whole, and especially those with the responsibility for funding and coordinating the research, need to look at discovery as a long-term, multiprong investment, which, in addition to fostering some digging expeditions to unearth the small gears, springs and levers, supports bigger coordinated efforts by multidisciplinary teams and brings them all back together to assemble the pieces into a functional machine.

\section{Acknowledgement}

The author would like to thank his coworkers Peggy Johnston and Alan Fix for their critical review of this manuscript.

Financial \& competing interests disclosure

The author has no relevant affliations or financial involvement with any organization or entity with a financial interest in or financial conflict with the subject matter or materials discussed in the manuscript. This includes employment, consultancies, honoraria, stock ownership or options, expert testimony, grants or patents received or pending, or royalties.

The author thanks Runa Musib for her expert editorial assistance. No other writing assistance was utilized in the production of this manuscript.
} 


\section{Bibliography}

1. Granich RM, Gilks CF, Dye C, De Cock KM, Williams BG: Universal voluntary HIV testing with immediate antiretroviral therapy as a strategy for elimination of HIV transmission: a mathematical model. Lancet 373(9657), 48-57 (2009).

2. Flynn NM, Forthal DN, Harro CD, Judson FN, Mayer KH, Para MF: Placebocontrolled phase 3 trial of a recombinant glycoprotein 120 vaccine to prevent HIV-1 infection. J. Infect. Dis. 191, 654-665 (2005).

3. Buchbinder SP, Mehrotra DV, Duerr A et al.: Efficacy assessment of a cell-mediated immunity HIV-1 vaccine (the Step Study): a double-blind, randomised, placebocontrolled, test-of-concept trial. Lancet 372, 1881-1893 (2008).

4. Fauci AS, Johnston MI, Dieffenbach CW et al.: HIV vaccine research: the way forward. Science 321, 530-532 (2008).

5. Keele BF, Giorgi EE, Salazar-Gonzalez JF et al.: Identification and characterization of transmitted and early founder virus envelopes in primary HIV-1 infection. Proc. Natl Acad. Sci. USA 105, 7552-7557 (2008).

6. Haase AT: Perils at mucosal front lines for HIV and SIV and their hosts. Nat. Rev. Immunol. 5, 783-792 (2005).

7. Pandrea I, Gaufin T, Brenchley JM et al.: Cutting edge: experimentally induced immune activation in natural hosts of simian immunodeficiency virus induces significant increases in viral replication and $\mathrm{CD} 4^{+} \mathrm{T}$ cell depletion. J. Immunol. 181, 6687-6691 (2008).

8. Gasper-Smith N, Crossman DM, Whitesides JF et al.: Induction of plasma (TRAIL), TNFR-2, Fas ligand, and plasma microparticles after human immunodeficiency virus type 1 (HIV-1) transmission: implications for HIV-1 vaccine design. J. Virol. 82, 7700-7710 (2008).

9. Gray ES, Moore PL, Choge IA et al.: Neutralizing antibody responses in acute human immunodeficiency virus type 1 subtype C infection. J. Virol. 81, 6187-6196 (2007).

10. Huisman W, Martina BE, Rimmelzwaan GF, Gruters RA, Osterhaus AD: Vaccine-induced enhancement of viral infections. Vaccine 27(4), 505-512 (2009).

11. Querec TD, Akondy RS, Lee EK et al.: Systems biology approach predicts immunogenicity of the yellow fever vaccine in humans. Nat. Immunol. 10(1), 116-125 (2009).

12. Silva AJ: The science of research: the principles underlying the discovery of cognitive and other biological mechanisms. J. Physiol. Paris 101, 203-213 (2007).

13. Robbins JB, Schneerson R: Future vaccine development at NICHD. Ann. NY Acad. Sci. 1038, 49-59 (2004).
14. Rappuoli R, Covacci A: Reverse vaccinology and genomics. Science 302, 602 (2003).

15. Muzzi A, Masignani V, Rappuoli R: The pan-genome: towards a knowledge-based discovery of novel targets for vaccines and antibacterials. Drug Discov. Today 12, 429-439 (2007).

16. Fellay J, Shianna KV, Ge D et al: A whole-genome association study of major determinants for host control of HIV-1. Science 317, 944-947 (2007).

17. Feyerabend P: Against Method. Thetford Press Limited, Norfolk, UK (1993).

\section{Website}

101. Joint United Nations Program on HIV/AIDS: 2008 report on the global AIDS epidemic. Geneva, Switzerland (2008). www.unaids.org/en/KnowledgeCentre/ HIVData/GlobalReport/2008/2008_ Global_report.asp

\section{Affiliation}

- Jorge Flores

Vaccine Research Program, Division of AIDS, National Institute of Allergy \& Infectious Diseases,

National Institutes of Health, Bethesda, MD 20892, USA

Tel.: +1 301435 3758;

Fax: +1 301402 3684;

jflores@niaid.nih.gov 